\title{
La investigación sobre los usos y los riesgos de los menores en el ciberespacio. Análisis metodológico ${ }^{1}$
}

\section{Research on Uses and Risks of Children and Adolescents in Cyberspace. Methodological Analysis}

Antonio García Jiménez. Universidad Rey Juan Carlos

María Cruz López de Ayala. Universidad Rey Juan Carlos

Carmen Gaona Pisonero. Universidad Rey Juan Carlos

Recibido: 17-II-2011 - Aceptado: 22-VI-2011

Resumen:

Se analizan las principales características metodológicas de la producción científica española sobre las prácticas online de los menores españoles. El trabajo se basa en una exhaustiva revisión bibliográfica de los artículos, libros, informes y comunicaciones publicados en la última década, con una doble dimensión que toma en consideración las iniciativas institucionales como las de carácter más académico. En la segunda parte del artículo, y tras una comparación con trabajos de carácter internacional, se analizan algunos de los planteamientos metodológicos más destacados y se focaliza la atención en los diseños metodológicos que implementen de forma interrelacionada tanto técnicas de investigación cuantitativas como cualitativas.

Palabras clave:

Internet, comunicación, adolescentes, riesgos, menores

Abstract:

The main methodological features of the Spanish scientific production about online practices of Spanish minors are analyzed. This work is based on a comprehensive literature review of articles, books, reports and papers published in the last decade with a double dimension that takes into account the institutional initiatives such as more academic nature. In the second part of the article, after a comparison with international research, we analyze some of the most important methodological approaches and focus attention on the methodological designs that implement both quantitative and qualitative research techniques in an interrelated form.

Keywords:

Internet, Communication, Adolescents, Risks, Minors

Este artículo es fruto del trabajo realizado en el marco del Proyecto Nacional de I+D+I "Análisis de uso y consumo de medios y redes sociales en Internet entre los adolescentes españoles. Características y prácticas de riesgo" (CSO2009-09577), Ministerio de Ciencia e Innovación. Secretaría de Estado de Investigación. Dirección General de Investigación y Gestión del Plan Nacional de I+D+I. 


\section{Introducción}

En los últimos diez años, hemos asistido en España a la proliferación de estudios que abordan la investigación empírica sobre los usos que los adolescentes realizan de Internet y los comportamientos asociados a ellos. Este interés por conocer las prácticas online de los menores no se circunscribe únicamente al ámbito académico, sino que diversas instituciones de iniciativa privada y pública, sensibles a las oportunidades y los peligros o riesgos que esconde la Red para los menores, han generado o patrocinado informes al respecto.

\section{Objetivos}

En este trabajo nos detendremos en las diversas investigaciones sobre esta cuestión generadas en España, con especial atención a aquellas iniciativas de carácter institucional que han sido las que han abarcado trabajos más ambiciosos desde el punto de vista del ámbito geográfico y representatividad de la muestra. Posteriormente, realizaremos un análisis descriptivo de las principales características metodológicas del conjunto de los estudios con el objetivo de identificar las principales tendencias al respecto, para, a continuación, establecer nuevos ejes temáticos sobre los que establecer nuevos diseños metodológicos. Por último, se realiza una reflexión sobre algunas de las cuestiones que se pueden analizar en este dominio.

Se pretende, con este estudio, orientar la reflexión entre la comunidad científica acerca del tratamiento temático y metodológico que está recibiendo este fenómeno entre los estudios empíricos en España con el objetivo de conocer sus puntos fuertes así como los sesgos, lagunas y carencias de que adolecen y a partir de ahí proponer un modelo metodológico que nos permita avanzar en el tema y sirva de referencia a otros investigadores.

\section{Hipótesis y contexto de investigación}

En el presente estudio, partimos de dos hipótesis de trabajo que han dirigido todo el esfuerzo realizado:

- Hipótesis 1.- Los trabajos empíricos que analizan las prácticas online de los adolescentes en el contexto español se enmarcan, en gran medida, en modelos metodológicos unidimensionales. En la medida en que estamos hablando de un fenómeno relativamente reciente que continúa consolidándose en su universalización entre la población adolescente, junto a la emergencia de servicios y aplicaciones novedosas como las redes sociales, también esperamos encontrar que la mayoría de los 
trabajos adoptan una metodología cuantitativa que busca describir el alcance social de este fenómeno, las pautas generales de uso y las principales variables que inciden en él.

- Hipótesis 2.- Esta segunda hipótesis hace referencia precisamente a la temática de los estudios relativos al tema. Debido a cuestiones como la caracterización social del colectivo a que se hace referencia -los menores, quienes son socialmente percibidos como seres inmaduros que están siendo socializados y que, por tanto, se encuentran en situación de especial vulnerabilidad-, el tratamiento que esperamos encontrar en los estudios revisados girarían en torno a la tríada temática uso/consumo/riesgo.

Para contrastar las hipótesis hemos hecho una exhaustiva revisión bibliográfica de los artículos, libros, informes y comunicaciones publicados en la última década que se plasma de forma resumida en la primera parte de este artículo. En los párrafos siguientes delimitamos los tres ejes conceptuales desde los que se parte:

1. Comunicación e infancia: este trabajo se enmarca dentro de los estudios que abordan la infancia y la juventud en su relación con la Comunicación. Se trata de un ámbito de investigación con gran relevancia a nivel internacional y que, a escala nacional, también presenta un evidente incremento en cuanto al número de estudios, trabajos y proyectos relacionados. Posiblemente, este hecho también se deba a la necesidad social y científica de analizar la interrelación entre los menores e Internet. Muchos de los temas tratados, fuera del dominio tecnológico, incorporan una visión preocupada por la protección de la infancia, de ahí que se traten aspectos como el impacto de la televisión en esta franja de edad, la regulación audiovisual en España, la publicidad o incluso la educación. Entre otros, podemos citar los siguientes autores: Gertrudix Manuel y Gertrudix, Felipe (2007); Núñez Ladevéze, L, Cantavella, J. y Vázquez Barrio, T. (2008), García Galera, Ma del Carmen (2008), Blanco, Ignacio y Römer, Max, (2010); Fuente Cobo, C. y Ruiz San Román, J. A. (2011).

2. Comunicación y riesgo: en nuestro contexto de análisis, el riesgo denota la posibilidad de que un estado de realidad indeseado pueda ocurrir como resultado del uso de las nuevas tecnologías. Ese riesgo no se produce y analiza desde una única magnitud, pongamos por caso desde la correlación existente entre las TIC y la dimensión psicológica en la que se habla, por ejemplo, del fenómeno de las adicciones a Internet para la población infantil y adolescente, sin olvidar nunca que lo que define la conducta como adictiva no es tanto la frecuencia con la que se realiza, sino la pérdida de control por parte del sujeto, el establecimiento de una relación de dependencia, la tolerancia y la interferencia con la vida cotidiana. Hemos establecido una correlación explicativa del riesgo asociado al uso de Inter- 
net, pero la incidencia negativa de las TIC en jóvenes y menores se puede extender a otras dimensiones como la física (trastornos de sueño), la familiar (disminución de prácticas y diálogo familiar) y la social (fracaso escolar, "ciberdelincuencia" -desde los piratas informáticos, a los distribuidores de pornografía infantil por Internet) entre otras más. Sin llegar a demonizar las prácticas en el ciberespacio, la importancia de establecer dinámicas lógicas de estas correlaciones es clara y evidente, pues una localización comprensiva de situaciones de riesgo en grupos poblacionales concretos supone un buen comienzo desde el que diseñar estrategias concretas que posibiliten eliminar o al menos reducir los riesgos de los menores en el ciberespacio. Por último señalar que este carácter multidimensional del riesgo que señalamos está presente tanto en su origen como en su estudio, por lo que encontramos diferentes enfoques para su comprensión: un enfoque psicológico, un enfoque antropológico y un enfoque sociológico (Ramírez, 2009).

3. Comunicación y tecnología: sin ser este el objeto de nuestro artículo, reproducimos algunos datos que nos ayuden a vislumbrar y comprender el alcance de las TIC en nuestra vida cotidiana española. De este modo tenemos que el 59\% de los hogares españoles dispone de conexión a Internet, una tasa de penetración relativamente baja en comparación con los países de la Unión Europea (INE 2010); dato este último que debemos correlacionar con el hecho de que sean los hogares en los que están presentes menores, principalmente mayores de 10 años, los que presentan tasas superiores de acceso a Internet frente a aquellos hogares formados exclusivamente por adultos. Siguiendo con datos significativos, el 87,3\% de los niños de 10 a 15 años utilizan Internet en España y el 96,3 \% de los jóvenes de 16 a 24 años (INE, 2010), porcentajes muy superiores a los del resto de la población en Europa. Pero en la reflexión sobre comunicación y tecnología no nos hemos de quedar en la mera recolección de datos, sino tras ver, a partir de éstos, la presencia real de Internet en este caso concreto de las vidas de los españoles, iniciemos nuestra comprensión y conocimiento de una Internet ubicua, personal, sistémica y geolocalizada que resignifica de forma veloz nuestro nivel comunicativo y relacional.

\section{Metodología}

En este apartado se examinan los aspectos más significativos de los planteamientos y la metodología de los estudios empíricos realizados en España que generan datos primarios sobre los comportamientos y actitudes de los adolescentes ante Internet. A tal efecto, se ha realizado una revisión bibliográfica de los artículos, libros, informes y comunicaciones publicados en España entre el año 2001 y diciembre de 2010 que abordan este tema e incluyen aspectos desde una mirada fundamentalmente comunicológica. El exa- 
men y reflexión sobre las metodologías y las prácticas investigadoras analizadas, así como de la experiencia de un proyecto similar restringido a la Comunidad de Madrid realizado anteriormente por parte del equipo que firma este artículo ${ }^{2}$, constituyen el punto de partida de una propuesta metodológica sobre el estudio de la relación de los adolescentes con Internet dentro del marco del proyecto nacional de investigación "Análisis de uso y consumo de medios y redes sociales en Internet entre los adolescentes españoles. Características y prácticas de riesgo" (CSO2009-09577), que es el motor del presente trabajo.

\subsection{Bases de datos y fuentes consultadas}

El rastreo y la recopilación de los documentos se ha realizado a partir de la base de datos ISOC (Ciencias Sociales y Humanidades), elaborada por el CSIC (Centro Superior de Investigaciones Científicas), que recoge fundamentalmente artículos de revistas científicas y de forma selectiva actas de congresos, series, compilaciones, informes y monografías. También se ha utilizado el portal de difusión de producción científica hispana Dialnet, que recopila artículos de revistas, artículos de obras colectivas, tesis y libros. Por último, además de emplear Google Académico, se ha llevado a cabo una revisión exhaustiva de los artículos publicados en las principales revistas de comunicación españolas, empleando como fórmula de búsqueda las mismas referencias bibliográficas de los documentos consultados. Los descriptores de la búsqueda han sido: Internet y adolescentes-menores-jóvenes.

Posteriormente, se han seleccionado aquellos estudios empíricos que toman como universo de estudio a los adolescentes españoles con edades comprendidas entre 12-17 años, aunque no se restrinjan a éstos. Otro criterio de inclusión en nuestra muestra fue que las unidades respondientes estuviesen constituidas por los mismos adolescentes, por lo que hemos desechado aquellos estudios que generan sus datos a partir de las informaciones de los padres o educadores en cuanto mediadores de la relación de los adolescentes con el ciberespacio. Respecto al ámbito geográfico abarcado, recogemos aquellos trabajos que se han realizado en el territorio español, pero también aquellos que se refieren a un área geográfica más restringida. Por último, aunque hemos incluido investigaciones que no planteaban como objeto de estudio principal o exclusivo el uso de Internet, se han descartado aquellas con un abordaje excesivamente marginal. Igualmente, hemos eliminado los trabajos que se limitan al estudio exclusivo de una herramienta/servicio específico disponible a través de Internet (blogs, comunidades virtuales, chat, fotolog o redes sociales).

Proyecto "Estudio sobre los usos de Internet entre los menores de la Comunidad Autónoma de Madrid. Riesgos y características" financiado por la Comunidad Autónoma de Madrid y la Universidad Rey Juan Carlos durante el año 2009. Es el precursor del Proyecto Nacional de I+D+I en el que se sitúa este artículo. 
Las revistas de comunicación consultadas han sido: Ámbitos: Revista Andaluza de Comunicación; Anàlisi. Quaderns de Comunicació i Cultura; Comunicación y Hombre; Comunicación y Pluralismo; Comunicación y Sociedad; Comunicar: Revista científica iberoamericana de comunicación y educación; Cuadernos de Información y Comunicación; Doxa Comunicación: Revista interdisciplinar de estudios de comunicación y ciencias sociales; Quaderns del CAC, Revista Científica de Información y Comunicación; Revista Latina de Comunicación Social; Sphera Pública: Revista de ciencias sociales y de la comunicación; Telos: Cuadernos de comunicación, tecnología y sociedad; Zer: Revista de Estudios de Comunicación. Se han consultado los números disponibles desde 2001 hasta la última revisión de artículos que se realizó el 15 de diciembre de 2010 .

Resulta conveniente advertir también que los artículos que se correspondían con un mismo proyecto y a una misma base muestral, finalmente, han sido recogidos en una referencia única.

\section{Iniciativas}

\subsection{Plano institucional}

En primer lugar, presentamos aquellos trabajos que tienen un origen más vinculado al plano institucional. Si bien muchos estudian diversas facetas del objeto de estudio analizado, podemos diferenciar las siguientes propuestas: 
Cuadro 1. Tipología de la producción científica española sobre las prácticas online de los menores españoles (plano institucional)

\begin{tabular}{|c|c|c|}
\hline CLASIFICACIÓN & AMBITO & EJE TEMÁTICO \\
\hline Estudios de carácter genérico & $\begin{array}{l}\text { Nacional } \\
\text { Psicología, Sociología y } \\
\text { Ciencias Comunicación }\end{array}$ & $\begin{array}{l}\text { Prácticas generales de uso de } \\
\text { las TIC por parte de menores } \\
\text { y jóvenes }\end{array}$ \\
\hline Estudios sobre riesgos y seguridad & $\begin{array}{l}\text { Nacional } \\
\text { Psicología, Sociología y } \\
\text { Ciencias Comunicación }\end{array}$ & $\begin{array}{l}\text { Contemporaneidad del riesgo } \\
\text { tecnológico }\end{array}$ \\
\hline Estudios sobre sickness e Internet & $\begin{array}{l}\text { Nacional y Local } \\
\text { Psicología }\end{array}$ & $\begin{array}{l}\text { Salud, adicciones y } \\
\text { ciberpatologías }\end{array}$ \\
\hline $\begin{array}{l}\text { Estudios de evaluación y } \\
\text { promoción }\end{array}$ & $\begin{array}{l}\text { Comunidades Autónomas } \\
\text { Carácter Institucional } \\
\text { (Observatorios de la Sociedad } \\
\text { de la Información) } \\
\text { Psicología, Sociología y } \\
\text { Ciencias Comunicación }\end{array}$ & $\begin{array}{l}\text { Evaluación sobre el impacto } \\
\text { de las TIC. } \\
\text { Promoción del Uso de las TIC } \\
\text { en educación y nueva cultura } \\
\text { audiovisual }\end{array}$ \\
\hline
\end{tabular}

Fuente: Elaboración propia.

A continuación se detallan algunas investigaciones relativas a la tipología establecida:

Estudios de carácter genérico. En 2006, el Observatorio Nacional de las Telecomunicación y la Sociedad de la Información (ONTSI), adscrito al Ministerio de Industria, Turismo y Comercio, publicó el informe Infancia y adolescencia en la Sociedad de la Información (Red.es, 2006). En el citado informe se analiza la relación de los menores de 18 años con las tecnologías de la información y comunicación, así como la relación de la presencia de niños en el hogar y las actitudes y usos de los adultos. Este trabajo se elaboró sobre los datos de la quinta oleada del panel trimestral de hogares Red.es realizado entre julio y septiembre de 2004, con una muestra representativa de 3178 hogares y 7423 individuos de la península y los dos archipiélagos.

Desde el año 2009 se han ido sumando nuevas iniciativas como las del Foro Generaciones Interactivas, el Observatorio de la Sociedad de la Información, la Fundación Pfizer y el Defensor del Pueblo (2010). El Foro Generaciones Interactivas es una organización sin ánimo de lucro fundada en 2008 por 
Telefónica, la Universidad de Navarra y la Organización Universitaria Interamericana (OUI) e integrada por profesionales de múltiples ámbitos (académico, docente, empresarial...) y nacionalidades cuyo objetivo es promover un uso responsable de las TIC entre los niños y jóvenes. El universo de estudio del trabajo La generación Interactiva en España. Niños y jóvenes ante las pantallas, publicado en noviembre de 2009, abarca el conjunto de alumnos de primaria, ESO y bachillerato en España, excluyendo Ceuta y Melilla. El estudio se basó en casi 13.000 encuestas realizadas a niños con edades comprendidas entre los 6 y los 18 años escolarizados en los ciclos educativos mencionados.

Situándonos ya a comienzos del año 2011, el Foro de Generaciones Interactivas ha publicado un nuevo informe titulado Menores y redes sociales, en el que se analiza el perfil de uso de las redes sociales de los niños y adolescentes españoles, poniéndolo en relación con el uso que hacen de otras pantallas (móviles, televisión y videojuegos) y otras variables como el acceso a la tecnología, su conocimiento, sus relaciones familiares, sus resultados escolares, y también con las valoraciones sobre los riesgos y las oportunidades de las pantallas. En el estudio fueron entrevistados 12.919 alumnos de primaria, ESO y Bachillerato procedentes de 78 colegios (55 públicos y 23 privados) de toda la geografía española, a excepción de Ceuta y Melilla. La recogida de datos se realizó mediante un cuestionario online al que los alumnos tuvieron acceso desde las aulas informáticas de su centro escolar. Se aplicó un muestreo bietápico, estratificado en la primera etapa con afijación proporcional según Comunidad Autónoma y titularidad (público o privado) y un muestreo aleatorio para la selección de los centros en la segunda etapa. La recogida de datos se realizó durante los meses de marzo a junio de 2009.

En el marco del programa del Observatorio de la Sociedad de la Información, desarrollado por el Instituto Nacional de Tecnologías de la Comunicación (INTECO) y que, a su vez, viene promovido por el Ministerio de Industria, Turismo y Comercio, se publicó el Estudio sobre hábitos seguros en el uso de las TIC por niños y adolescentes y e-confianza de sus padres. En este estudio de carácter nacional vienen recogidos hábitos de uso de las TICs por parte de los menores, así como sus conocimientos sobre la seguridad de la información y herramientas utilizadas. El estudio se basa en 1.200 encuestas, de las cuales la mitad corresponde a los menores con edades comprendidas entre los 10 y 16 años y el resto a sus padres o tutores legales, lo que permite contrastar la visión de ambos colectivos.

Por otra parte, en septiembre de 2009, TNS Demoscopia y la Fundación Pfizer presentaron en España "La Juventud y las Redes Sociales en Internet". Este informe analiza actitudes y usos de las redes sociales entre los adolescentes y presenta algunos datos interesantes sobre el papel mediador de padres y madres. El estudio se realizó mediante entrevista telefónica a una muestra representativa de 1.000 
adolescentes españoles de entre once y veinte años y sus padres y madres. Utilizó un muestreo polietápico, estratificado y aleatorio para la selección de los hogares con los que se contacta, y, ya a escala de hogar, con aplicación de cuotas de sexo y edad para determinar la muestra de jóvenes, y de una cuota exclusivamente de género para concretar el progenitor que se entrevista (dos entrevistas por hogar, hijo/a y uno de sus progenitores).

- $\quad$ Estudios que analizan los riesgos y la seguridad. Cabe mencionar, en primer lugar, a la ONG Protégeles, que fue pionera en este interés por los peligros que acechan a los menores en la Red y elaboró sendos informes en 2002, junto con la ONG ACPI (Acción Contra la Pornografía Infantil), y en 2004, con un trabajo elaborado para el Defensor del Menor. El informe Seguridad Infantil y Costumbres de los Menores en Internet (2002) se realizó sobre un total de 4000 encuestas entre menores de 10 a 17 años, de ambos sexos, obtenida en centros escolares, en cibercentros, en concentraciones temporales de menores, a escala de calle, así como a través de portales infantiles en Internet. Por otra parte, Cibercentros y Seguridad Infantil en Internet (2004) es un trabajo basado en una encuesta realizada a una muestra de 1.000 menores de ambos sexos, de edades comprendidas entre los 11 y los 17 años, obtenida en centros escolares y en cibercentros.

Más recientemente, el Defensor del Pueblo publicó el informe Programación y contenidos de la televisión e Internet: la opinión de los menores sobre la protección de sus derechos, que incluye entre sus objetivos, el análisis de los riesgos del uso de Internet. Este trabajo se basa en una encuesta realizada a una muestra 3.219 adolescentes, de entre 12 y 18 años, que cursaban Educación Secundaria Obligatoria (ESO), Bachillerato y Formación Profesional (FP) en el curso 2009/2010, en 150 centros de titularidad pública y concertada de todo el territorio español.

Estudios sobre sickness e Internet. Desde el ámbito sanitario, el Instituto de Adicciones Madrid Salud publicó en 2008 el Estudio de uso problemático de las tecnologías de la información, la comunicación y el juego entre adolescentes y jóvenes de la ciudad de Madrid. Este trabajo pretende conocer en profundidad los aspectos cualitativos del uso de las tecnologías de la información y la comunicación, además de aproximarse a la cuantificación de las prácticas discursivas que aparezcan como más problemáticas. El estudio utiliza una metodología mixta, cualitativa y cuantitativa. Para el acercamiento a las prácticas de los adolescentes madrileños se utilizan encuestas en hogares (padres e hijos). En total se entrevistó a 227 adolescentes de 12 a 18 años (118 hombres y 109 mujeres).

Estudios de evaluación y promoción. El informe La transmisión de valores a los menores, realizado durante 2007 para el Defensor del Pueblo del País Vasco -Institución Ararteko-, en su propósito de 
analizar el grado en que los menores asumen una serie de valores básicos, así como la fuerza o incidencia que tienen los diferentes agentes sociales en la transmisión o construcción de dichos valores, recopila algunos datos sobre el consumo de Internet entre los adolescentes vascos. La investigación se realiza utilizando metodologías mixtas que incluyen análisis documental, grupos de discusión y encuesta. La muestra del estudio, de carácter cuantitativo, incluye a 1.829 estudiantes del País Vasco, 762 de ESO, en 25 centros educativos públicos y privados.

También el Observatorio de la Infancia en Andalucía publicó dos informes relativos al tema en 2008 y 2010. El primero de ellos, Nuevas Tecnologías e Infancia y Adolescencia 2008, se sustentó en datos cualitativos procedentes de 6 grupos focales con 40 niños y adolescentes de 9 a 17 años (22 mujeres y 18 hombres) en la Comunidad Autónoma de Andalucía. Aunque incluye apartados dedicados a datos cualitativos y cuantitativos, únicamente genera datos primarios con grupos de discusión ya que en la información cuantitativa proviene de los resultados de Encuesta de Hogares sobre equipamiento y uso de Tecnologías de la Información (INE). El segundo informe, Actividades y usos de TIC entre chicos y chicas de Andalucía. Informe 2010, se elaboró sobre datos cuantitativos generados mediante encuesta, con una muestra de 1.212 chicos y chicas de entre 9 y 16 años. El trabajo de campo se realizó durante marzo de 2009 en 54 centros de primaria y secundaria de titularidad pública y privada-concertada de la Comunidad Autónoma de Andalucía.

\subsection{Plano académico}

Desde un ámbito con mayor presencia académica podemos encontrar acercamientos empíricos al fenómeno de los usos y los comportamientos de riesgos asociados a la Red desde diferentes disciplinas y campos de estudio, destacando las aproximaciones desde los estudios de comunicación, la sociología y la psicología. Frente al formato más homogéneo de informe descriptivo-distributivo que generan las iniciativas de instituciones no académicas, entre las investigaciones originadas en el ámbito universitario se observa una mayor diversidad. Así, en la producción académica son frecuentes los acercamientos cuantitativos meramente descriptivos y, probablemente debido a limitaciones presupuestarias, no resulta extraña la utilización de muestras no representativas (Navarro, 2003; Graner et al, 2006 y 2007; Hernández, 2006) y referidas a ámbitos geográficos restringidos a una provincia o comunidad autónoma; no son muchos los estudios que utilizan muestras nacionales como los de Aranda y otros (2009 y 2010) y Sánchez Burón y otros (2010). Así pues, presentamos una clasificación de los diferentes trabajos, recogida en el siguiente cuadro: 
Cuadro 2. Tipología de la producción científica española sobre las prácticas online de los menores españoles (plano académico)

\begin{tabular}{|lll|}
\hline CLASIFICACIÓN & ÁMBITO & EJE TEMÁTICO \\
\hline Estudios Kids-grading nacional & Nacional & $\begin{array}{l}\text { Prácticas comunicativas: ocio } \\
\text { digital, uso redes sociales }\end{array}$ \\
& $\begin{array}{l}\text { Cuantitativos/descriptivos } \\
\text { Psicología }\end{array}$ & \\
\hline Comunicación Audiovisual & \\
\hline Estudios prospectivos & $1 / 3$ Nacional 2/3 Autonómicos & Riesgos \\
& y local & Agentes socializadores de \\
& Cualitativos o cuantitativos & Internet: familia, grupo, escuela. \\
& Psicología & Hábitos de uso de Internet \\
\hline País Vasco/ Comunidad de Madrid & Uso de medios. \\
& Madrid, Granada y Cáceres & Riesgos \\
& Sociología, Psicología, & Uso abusivo de las TIC y el juego \\
& Comunicación y Ciencias de la Salud & \\
& Carácter mixto (cualitativo y & \\
& cuantitativo) y triangulación & \\
& secuencial & \\
\hline
\end{tabular}

Fuente: Elaboración propia.

Estudios Kids-grading nacionales. Dentro de estos trabajos con cobertura nacional situamos un proyecto realizado por un equipo de profesores de la Universitat Oberta de Catalunya: Transformemos el ocio digital: un proyecto de socialización en el tiempo libre. Con el propósito de estudiar las prácticas comunicativas que realizan los adolescentes, se realizó una encuesta representativa de ámbito nacional (exceptuando Canarias, Ceuta y Melilla) en la que fueron preguntados tanto padres como hijos. La muestra estuvo formada por 2.054 adolescentes con edades comprendidas entre los 12 y los 18 años que fueron entrevistados a lo largo de la segunda quincena de marzo de 2009. El tipo de muestreo utilizado fue polietápico, seleccionando primero municipios y posteriormente hogares e individuos. El error muestral para el conjunto de la muestra fue del 2,16\% y se eleva al 5\% para los resultados de la Comunidad Autónoma de Andalucía y Cataluña, siendo mucho más altos para el resto de comunidades que conforman el Estado Español (Aranda, 2009 y 2010). 
Por otra parte, un grupo de profesores del Departamento de Psicología de la Universidad Camilo José Cela ha centrado el foco de atención en el uso que hacen los adolescentes de las redes sociales. En junio de 2009 publicaron un primer informe cuyo universo de estudio se circunscribe a la población escolarizada en educación secundaria (ESO) de la Comunidad de Madrid. En total se entrevistó a 1.095 alumnos de seis centros de ESO, con lo que el error muestral que se refiere alcanzaría el +/- 3 para un nivel de confianza del 95,5\% (Sanchez Burón et al. 2009). Más recientemente y con el objetivo de realizar un análisis comparativo entre las 17 comunidades autónomas, este estudio de carácter principalmente descriptivo se amplió al conjunto del territorio español, recogiendo una muestra de 6.798 adolescentes de entre 11 y 20 años, escolarizados en Educación Secundaria obligatoria y primer curso de bachillerato de 44 centros públicos, privados y concertados de las 17 comunidades autónomas (Sanchez Burón et al. 2010).

- Estudios prospectivos. En el ámbito académico también son frecuentes los estudios cualitativos de ámbito geográfico restringido. A lo largo de 2001, Albero y otros (2002) llevaron a cabo una investigación de carácter exploratorio que pretendía advertir cómo la familia, la cultura de grupo, la escuela, y la experiencia previa con otros medios de comunicación orientan el uso de Internet entre los adolescentes. En el estudio se combinó el uso de la entrevista individual, y entrevista en grupo, con la observación participante con los sujetos conectados en solitario o con amigos y con entrevistas a padres y dos profesores. Los 80 adolescentes de entre 12 y 17 años que participaron en el estudio provenían de 20 colegios de Barcelona y sus alrededores. En el VIII Congreso de Estudios Extremeño, Madruga y otros (2005) presentaron una comunicación en la que se presenta una investigación cualitativa, con grupos de discusión, cuyo objetivo era analizar los hábitos de vida relacionados con la salud, así como el uso de las nuevas tecnologías (Internet) en adolescentes extremeños. El universo de estudio estaba conformado por chicos de entre 12 y 14 años reclutados en colegios de la ciudad de Cáceres.

- $\quad$ Estudios prospectivos cuantitativos. Junto a estos estudios descriptivos, en el ámbito de la Academia también coexisten investigaciones de carácter explicativo que buscan establecer modelos predictivos del comportamiento online de los adolescentes. Una gran parte de estos estudios -provengan de la iniciativa pública o privada- recurren a metodologías cuantitativas, y más concretamente a la encuesta, en un intento de cuantificar y describir los usos online de los adolescentes en este proceso de generalización del acceso a Internet de la sociedad española, en general, y de este grupo, en particular. Los últimos años, 2009 y 2010, han sido especialmente prolíficos en la producción de trabajos empíricos y, en particular, en la generación de datos cuantitativos. A lo largo de estos dos años hemos 
contabilizado hasta 13 trabajos publicados y en todos los casos emplean la encuesta como técnica paradigmática que representa esta perspectiva distributiva.

Estos estudios de carácter cuantitativo pueden clasificarse en dos grupos. Un primer grupo de estudios trabaja con muestras no probabilísticas y explicitan, en términos generales, su carácter exploratorio. Estas investigaciones utilizan muestras intencionales o de conveniencia basadas en un número muy limitado de centros educativos, de uno a tres. El segundo grupo está formado por trabajos que emplean muestras probabilísticas y muestreos polietápicos por conglomerados y/o estratificados, si bien, salvo excepciones, no se explicitan todas las características del diseño, o al menos las ponderaciones derivadas de éste.

En cuanto al área geográfica, algo menos de una tercera parte de los estudios que recurren a la encuesta cubren todo el territorio nacional; el resto abordan este fenómeno en diferentes comunidades autónomas: País Vasco, Comunidad de Madrid, Andalucía, Islas Baleares y Región de Murcia. Otros ámbitos geográficos representados son: ciudad de Córdoba, Barcelona y alrededores, provincia de Barcelona, ciudad de Cáceres, ciudad de Madrid y ciudad de Gerona.

En general, estos estudios recopilan datos relativos a los hábitos de uso de Internet por parte de los adolescentes, con preguntas acerca de los servicios utilizados, el lugar de acceso, el tiempo de uso, etc. Por otra parte, tal y como ya avanzábamos anteriormente, una parte importante analiza el fenómeno desde la perspectiva de los riesgos (ACPI-Protégeles, 2002; Defensor del Menor y ACPI-Protégeles, 2004; Hernández, 2006; Castellana et al., 2007; Garitaonandia y Garmendia, 2007; Torrecilla, 2008; Observatorio de la Seguridad en la Información, 2009; García Jiménez, 2010b; Defensor del Pueblo, 2010; Sánchez, 2010). También hemos detectado esta misma preocupación en trabajos que, desde el área de la psicología, o bien están orientados a los efectos del uso de Internet sobre determinadas conductas, por ejemplo, sobre el rendimiento académico (Conde, Ruiz y Torres-Lana, 2010) o, por el contrario, buscan establecer modelos predictivos del uso abusivo de Internet de acuerdo con el perfil psicológico de los menores (Viñas, 2009).

Además, en muchos de estos trabajos, se representa a los menores como un colectivo socialmente vulnerable al que la sociedad tiene la obligación de proteger, lo que puede dificultar el acercamiento objetivo al análisis de la relación de los menores con Internet sin caer en el prejuicio (Callejo, 2004). Este hecho puede ser la causa de la proliferación de documentos, de diferente índole, que adoptan un discurso normativo más que científico en el análisis de la relación de los menores con las tecnologías de la información y comunicación. 
Esta predisposición se hace más patente, si cabe, en el caso de la encuesta. Resulta ya clásica la denuncia desde ciertas escuelas cualitativistas de la propensión de esta técnica a analizar un "mundo ficticio e inexistente construido por el observador científico" en la medida que impone la formulación, no solo de la preguntas, sino también de las categorías de respuesta a los sujetos analizados (Shütz, 1964: 15). Esta deficiencia se une a los prejuicios que acompañan la investigación sobre los menores y se manifiesta en la imposición de las preocupaciones de los adultos a los niños, pues, como indica Callejo (2004), es la imposición de las preocupaciones de las instituciones que investigan a los encuestados.

Mucho menos común que los anteriores resultan los estudios que hacen uso de metodologías cualitativas, aproximándose a este fenómeno desde la mirada y la voz de los propios adolescentes. Menos de una cuarta parte de los estudios contemplados en la revisión utilizan exclusivamente metodologías cualitativas. Dentro de la perspectiva estructural o cualitativa, la práctica más comúnmente utilizada es el grupo de discusión y los grupos focales. En la mitad de los casos, la investigación adquiere una dimensión nacional (Garitaonandia y Garmendia, 2007; Espinar y González, 2008); la otra mitad se refiere a territorios más restringidos, como Andalucía y Extremadura, (Observatorio de la Infancia de Andalucía, 2010; Madruga el al., 2007). Por otra parte, dos de los estudios identificados combinan la entrevista con la observación participante (Albero, 2002; Gil et al., 2003).

- $\quad$ Estudios Kids-grading local. Todavía es menos frecuente la aproximación al tema desde metodologías mixtas. Además, ninguno de los escasos trabajos que incorporan esta mirada y combinan el uso de técnicas de investigación cualitativa y cuantitativa cubre la totalidad de España. Dos de ellos se circunscriben al País Vasco (Ramírez de la Piscina, 2006 y Ararteko, 2009), otro a la Comunidad de Madrid y la provincia de Granada (Rubio, 2009), un cuarto a la Comunidad de Madrid exclusivamente (García, 2010b) y, por último, otro se refiere a la ciudad de Madrid (Torrecilla, 2009).

En cuanto a la forma de articular las metodologías cuantitativas y cualitativas, el estudio realizado en el País Vasco por Ramírez de la Piscina (2006) utiliza una triangulación secuencial y produce informes segregados, por lo que carece de la integración posterior que permitiría alcanzar una visión holística del fenómeno. Este trabajo aborda el uso de medios en general, tratándose el uso de Internet de forma muy sucinta. El informe de la Institución Ararteko (2009), dirigido por Javier Elzo, también genera informes independientes para las encuestas realizadas en los diferentes niveles educativos y para los grupos de discusión.

Por su parte, Torrecilla (2009) dirige un estudio realizado en la ciudad de Madrid publicado por Instituto de Adicciones de Madrid Salud, en el que se articula de forma más interactiva técnicas 
cualitativas y cuantitativas, sirviendo los resultados de los grupos de discusión como estudio exploratorio para determinar las hipótesis de investigación y poder elaborar el cuestionario, además de indagar en las percepciones sobre el problema y las motivaciones profundas que conducen a un uso abusivo de las TIC y del juego. No obstante, los integrantes de estos grupos de discusión son jóvenes (19-25 años) que han registrado un uso excesivo o no de tecnologías de la información, comunicación y juego en un cuestionario previamente realizado.

En el Estudio sobre los usos de Internet entre los menores de la Comunidad de Madrid. Riesgos y características (García, 2010b), que antecede y sirve de referente de la investigación nacional sobre la que gira este artículo, se buscaba la interconexión entre el uso de técnicas cualitativas y cuantitativas con el objetivo de forjar una visión integrada entre la descripción y explicación de los usos de Internet y la comprensión del fenómeno investigado. En este trabajo, los grupos de discusión fueron realizados antes y después de la encuesta, de manera que ayudaron a la formulación del cuestionario a la par que sirvieron para interpretar los resultados del estudio cuantitativo.

Por su parte, el estudio dirigido por Ma Ángeles Rubio también muestra un alto grado de interpenetración entre el análisis cualitativo y cuantitativo. Las entrevistas en profundidad, junto con el análisis de casos, han servido para definir patrones de consumo que después sirven como referencia en el diseño del cuestionario. Paralelamente, el cuestionario sirvió "para contabilizar la incidencia de Internet y sus contenidos, pero sobre todo, confirmar la interpretación y aplicación que esta población hace del medio" (Rubio, 2009: 10).

Desde un punto de vista general, cabe destacar la generación de datos descriptivos relativos a los usos que los menores hacen de Internet mediante el empleo de los grupos de discusión y grupos focales. En este sentido, sobresale la descripción de porcentajes sobre lugares de acceso, franjas horarias de conexión y tipos de uso que los adolescentes participantes en el grupo de discusión hacen de Internet en uno de los estudios ubicados en la ciudad de Cáceres (Madruga et al., 2007). El tratamiento de estas prácticas cualitativas se justifica o por el carácter exploratorio que se atribuye a estas investigaciones o por las restricciones presupuestarias, como así se expresa en el trabajo integrado dentro del proyecto EUKids Online en España (Garitaonandia y Garmendia, 2007). En este caso, y en el marco de la financiación europea, la investigación se ha continuado ya con una fuerte aproximación cuantitativa (Garmendia, M. et al., 2011). 


\subsection{Breve comparativa internacional}

En un nivel europeo, tal y como apuntan Livingstone y Haddon (2008), las investigaciones empíricas relacionadas con las experiencias en línea de los niños presentan las siguientes características: a) predominan las de carácter nacional, frente a los estudios multinacionales; b) los investigadores proceden fundamentalmente de las áreas de educación, información o psicología; c) en gran medida, suelen estar encargadas por agencias comerciales o las de protección/cuidado de los niños; d) hay menos estudios cualitativos o con multi-métodos, por lo que existe un menor conocimiento de las propias experiencias de los menores y sus percepciones; e) la mayor parte están aplicados a adolescentes; f) en general, se necesita investigaciones transnacionales, multidisciplinares, que empleen más de un método, contextuales y longitudinales.

En términos globales, se puede confirmar una mayor presencia de estudios cuantitativos. De entre los muchos trabajos existentes de esta naturaleza, a título ilustrativo, nos detenemos en varios. Por un lado, el estudio de Aslanidou y Menexes (2008) tiene como objetivo la detección de patrones sistemáticos de comportamiento en relación con el uso, así como aquellos factores que pueden influir dentro del ambiente familiar en el hogar en la relación entre los jóvenes e Internet. También pretende desarrollar una tipología de estudiantes en función de los usos y comportamiento y estudiar los posibles efectos de la estratificación geográfica, social, de género y por edad. A tal efecto, aplicaron una cuestionario (autoadministrado y estructurado) procedente del European Research Project Mediappro (2006), que incluía 39 preguntas cerradas multi-item con diferentes opciones (múltiples opciones, elecciones binarias, de respuesta múltiple, y con listas de orden) y una pregunta abierta (¿qué significa Internet para ti?).

Por su parte, uno de los informes del Pew Research Center's Internet \& American Life Project (Lenhart et al., 2010), que trata de comparar el uso de Internet y los medios sociales entre los adolescentes y los adultos jóvenes, también usa una encuesta telefónica, que se lleva a cabo entre junio y septiembre de 2009, realizada a una muestra (en lo que afecta a los más jóvenes) de 800 adolescentes entre los 12 y los 17 años. El estudio de Siomos y otros autores (2008), centrado en la adicción, también focaliza la atención en estudiantes adolescentes entre los 12 y los 18 años. La muestra estaba compuesta por 2.200 estudiantes, fue reclutada de 120 clases entre 85 colegios en Thessaly (Grecia), con una selección estratificada aleatoria. En este caso, los estudiantes completaron el YDQ ((Diagnostic Questionnaire for Internet Addiction).

Por otra parte, en el trabajo de Chai y otros (2009), que se centra en el uso de la privacidad, más concretamente, en los factores que influyen en comportamiento a la hora de compartir información privada, se utilizó una encuesta de 285 preadolescentes de 11 a 14 años (sobre 400 solicitados). Con un cuestionario estructurado, emplearon la técnica partial least square que pretende maximizar la varianza a partir de las 
variables dependientes explicadas por las variables independientes en lugar de reproducir la matriz de covarianza empírica. No obstante, y a pesar de que la mayoría de los esfuerzos en este terreno están dirigidos a técnicas cuantitativas, también se pueden encontrar algunos trabajos en clave cualitativa, como el que desarrollan Hundley, H. L. and Shyles (2010).

\subsection{Delimitación de nuevos enfoques metodológicos}

Si bien nuestra perspectiva se sitúa más en el ámbito comunicológico que sociológico, coincidimos en gran medida (en el contexto de la American Sociological Association) con la propuesta metodológica de Michael Burawoy, la "sociología pública" que básicamente promueve tres principios básicos: 1) no existe un método ideal, sino que toda metodología bien fundamentada es válida; 2) la ciencia debe estar orientada hacia problemas y por último, 3) todo análisis y reflexión científica debe de integrar los efectos del contexto social, así como los efectos de campo. De alguna manera, Burawoy nos vuelve a dirigir hacia una ciencia social reflexiva, una perspectiva ya iniciada y seguida por otros científicos sociales como Pierre Bourdieu y Loïc Wacquant. Sobre la base de estas tres premisas, pretendemos reflexionar sobre algunas interrogantes metodológicas que surgen en los trabajos empíricos relacionados con nuestro objeto de estudio, tras la pertinente revisión bibliográfica, y que nos propusimos enfrentar en el trabajo Comunicación y comportamiento en el ciberespacio (García, 2010b).

Entendemos que no existe el método ideal. En un intento de buscar un autor neutral al que no podamos etiquetar de forma rápida y directa con un paradigma teórico, retomamos a uno de los grandes literarios del siglo XX, Jorge Luis Borges, quien muy acertadamente alude a las cuestiones aquí tratadas. En cierta medida, y sin situarnos en el relativismo metodológico, podría afirmarse que toda metodología bien fundamentada es válida. En realidad, cualquier método no debe de desviarse nunca del rigor de la ciencia, sin que este rigor sea concebido cartográficamente. Borges (1981: 142-156) alerta sobre este peligro al evocar el Arte de la Cartografía, que en sus orígenes intentaba reproducir hito a hito la realidad del paisaje, obteniendo como resultado aquellos primeros gigantescos y monumentales mapas. De aquellos primeros mapas la cartografía evolucionó hasta reproducir esa exactitud cartográfica de la realidad. En el caso de las ciencias sociales hablaríamos del Arte de las Estadísticas en la reproducción de la realidad social.

En modo alguno debe interpretarse esto como el cuestionamiento de la validez del rigor científico en la implementación de técnicas cuantitativas, todo lo contrario. Simplemente, hacemos especial hincapié en la idea de no pretender reproducir la realidad tal cual es y en todas sus dimensiones, puesto que es tan compleja que un modelo que diera cuenta de todos sus elementos sería inmanejable. Lo operativo es simplificar 
la realidad descrita, de ahí la practicidad en el tamaño muestral, sin que esto nos haga creer que nuestro rigor científico nos ha conducido a analizar a toda la población menor de edad.

En segundo lugar, tomamos la idea de la ciencia orientada a problemas y la derivamos a la problematización de los fenómenos sociales. En efecto, hemos detectado la tendencia a considerar el uso y consumo de las TIC (de medios, redes sociales en Internet, videojuegos, etc.) por parte de la población infantil y adolescente como un problema. Hay toda una corriente de opinión que se inclina a considerar negativamente determinadas prácticas digitales, como pueden ser las relacionadas con los videojuegos, relacionándolas con el aislamiento social, las adicciones, el desarrollo de una personalidad violenta, etc. En esta misma línea estaría la tesis de Iván Rodríguez Pascual, quien denuncia la excesiva "perspectiva adultocéntrica" de los investigadores desde la que se diseñan estos trabajos. En efecto, esta tendencia a problematizar los usos digitales está presente en una proporción considerable de los proyectos analizados. Las investigaciones partirían no tanto de la idea de estudiar una realidad a través de experiencias concretas y datos empíricos, sino más bien desde prejuicios que inciden en la vulnerabilidad, que tratan al adolescente o al menor más como una víctima que como un usuario (que como cualquier otro usuario también se puede enfrentar a riesgos).

En este sentido, en la investigación mencionada Comunicación y comportamiento en el ciberespacio (García, 2010b) fue de vital importancia los tres grupos de discusión que realizamos previos a la elaboración del cuestionario que vertebró nuestra encuesta. En éstos no solamente tuvimos una aproximación socioantropológica directa con los menores y adolescentes como auténticos ciudadanos-usuarios de las TIC, sino que nos ayudaron a evitar equívocas concepciones sobre las nuevas relaciones personales que establecen. A modo de ejemplo, reformulamos nuestro concepto "quedar con desconocidos", término del que partíamos y que tuvimos que variar en el cuestionario final, por haber una gran distancia semántica en la interpretación de "desconocidos" de los investigadores, y el valor de proximidad y confianza que otorgan los menores y adolescentes a aquellos "nuevos amigos" que hacen por el espacio virtual.

Sin desdeñar los posibles riegos e impactos negativos, también son relevantes los estudios que destacan que la relación de niños y jóvenes con las TIC repercute positivamente en sus procesos de socialización. De entre estos trabajos nos remitimos al de Olson (2007), que se centra en el consumo infantil y juvenil de los videojuegos. Esta autora defiende que la práctica de los videojuegos reproduce un contexto propiciador de un proceso de desarrollo infantil normal. De hecho, tal y como se constató, niños y adolescentes citan el uso de los videojuegos como una forma de socialización, ya que están entre los principales temas de conversación. De igual modo, se demuestra que el grado de habilidad de un niño cuando utiliza una consola también puede determinar su posición jerárquica dentro de su grupo de amigos. 
No podemos olvidar que el desafío y la competición suponen uno de los mayores atractivos de los videojuegos, tal y como declaran en relación con las motivaciones a la hora de jugar. A los jóvenes les gusta vencer no solo a los ordenadores, a los programas, sino también a otros jugadores. Y en algunos casos, el éxito de un joven en los videojuegos compensa sus dificultades en otras áreas, como la escuela o como las que tienen lugar en determinados contextos sociales. Desde esta perspectiva, también los juegos "multiplayer" pueden ser muy importantes en los procesos de socialización ya que ponen a los sujetos en la tesitura de desempeñar distintos roles (liderazgo, etc.). Otras consecuencias positivas de la práctica de los videojuegos se centran en la posibilidad de establecer relaciones personales orientadas al aprendizaje, el desarrollo de amistades online, etc. No cabe duda de la importancia de los factores emocionales que motivan el uso de las consolas: regulación de las emociones, sensación de influencia y control, superación de desafíos personales o expresión de la creatividad, entre otros.

Con la exposición anterior no defendemos la correlación única entre práctica de videojuegos y, por un lado, desarrollo de la personalidad y, por otro, acercamiento a procesos de socialización, ambos óptimos e ideales. Nuestro objetivo se basa más bien en alertar sobre la tendencia a demonizar la relación entre las TIC y la población infantil/juvenil, resaltando por ejemplo las posibilidades de adicción. A modo de puntualización metodológica, no olvidemos que ambas perspectivas se basan en investigaciones que han concluido en probabilidades empíricas, y en ningún modo se han detectado probabilidades lógicas que ofrezcan un tratamiento sistémico que merece la complejidad de estos nuevos fenómenos comunicativos.

En cualquier caso, la tendencia mayoritaria queda manifiesta en los siguientes estudios en los que se equipara el alto consumo de las TIC con conductas adictivas: el uso compulsivo de Internet (Greenfield, 1999a, 1999b; Meerke, Van den Eijden y Garretsen, 2006), la adicción a los ordenadores (Young 1996, 1998; Charlton, 2002; Charlton \& Danforth, 2007), el uso patológico de Internet (Davis, 2001), o problemático (Aboujaoude, Koran, Gamel, Large y Serpe, 2006; Caplan, 2003); en el contexto español también hay estudios sobre la adicción a las nuevas tecnologías de (Muñoz-Rivas, Navarro y Ortega, 2003; Viñas, Villar, Caparrós, Perez, Corrella, 2002; Echeburúa, 2009).

Es interesante ver que, de estos trabajos mencionados - prácticamente todos ellos elaborados desde una óptica psicologista-, las tasas de personas con problemas de adicción o uso excesivo varían de forma considerable entre el $6 \%$ y el 66\%, atendiendo a si las muestras son auto-seleccionadas o no (Sánchez-Carbonell y otros, 2008). Sobre esta correlación directa establecida, recordemos que lo que realmente define la conducta como adictiva no es tanto la frecuencia con la que se realiza, sino la pérdida de control por parte del sujeto, el establecimiento de una relación de dependencia, y su interferencia en la vida cotidiana. 
No obstante, a modo de defensa de los planteamientos metodológicos que imperan en las ciencias sociales, es pertinente reconocer la dificultad que entraña advertir este fenómeno mediante un simple cuestionario. Sí que es posible insertar en un cuestionario, tal y como hicimos en nuestra investigación, preguntas desde las que se registren síntomas de una adicción a Internet, síntomas de abstinencia físicos y psicológicos: alteraciones del humor, tristeza, irritabilidad, impaciencia, inquietud, ansiedad. O incluso factores relacionados con trastornos fisiológicos, que implican menos problemas a la hora de ser tenidos en consideración en el cuestionario, pero que deben ser contrastados puesto que pueden ser sintomatología de otros trastornos y enfermedades.

En otras ocasiones, también se sugiere que un alto consumo de Internet acrecienta la limitación de las formas de diversión y ocio, reduciendo las relaciones sociales y aumentando el aislamiento social. Sin embargo, en el último Informe de la Fundación Kaiser (2009) se apunta en una dirección divergente. En este sentido, no se detecta una relación directa, en las entrevistas realizadas, entre tiempo de exposición con la exclusión social, o incluso la actividad física. Sí se aprecian diferencias, pero no significativas, en los siguientes aspectos: bajo rendimiento académico, las relaciones parentales, niveles de infelicidad. Ante estos datos cabría hacer un replanteamiento y hablar de nuevos modelos de socialización en el que confluyen tanto dinámicas positivas como negativas, nuevos modelos de vida y relación social en el marco del "individualismo en red".

Finalmente, como decíamos al principio de este epígrafe, cuentan los efectos del contexto social así como los efectos del campo. Dentro de esos nuevos procesos de socialización en relación con las TIC y la población infantil/juvenil, cabe también hablar de nuevas formas de comunicación, fundamentalmente visual, tal y como señala Llorca (2009), entre otros. En efecto, las nuevas tecnologías implican nuevas formas de ver, diferentes imaginarios, en definitiva, debemos "pensar en los menores, no como consumidores o aprendices, sino como constructores e intérpretes de significados".

\section{Conclusiones y discusión}

La revisión de la metodología utilizada por los estudios que abordan el uso adolescente de Internet ha permitido constatar diversos hechos:

1. La prevalencia de modelos metodológicos unidimensionales, ya sean cuantitativos o cualitativos, como forma de acercarse a este objeto de estudio. Dentro de estos modelos, la perspectiva cuantitativa es la más frecuente y casi única si nos circunscribimos a las iniciativas institucionales. La encuesta 
estadística y los grupos de discusión son las técnicas/prácticas más usadas, generalmente de manera exclusiva. De esta forma, queda constatada la primera hipótesis de nuestro trabajo. Por último, es preciso señalar que los estudios revisados en determinadas ocasiones carecen de una descripción clara y extensa de la metodología seguida en el proceso de investigación que explique las decisiones relativas al proceso de selección de la metodología o la descripción del proceso de obtención/producción de datos. Además debiéramos añadir que, en muchos casos, no aportan un marco teórico explícito o no se profundiza en el mismo.

2. De igual modo, se confirma que el tratamiento básico de los estudios revisados giran alrededor de los temas uso/consumo/riesgo. Ha quedado claro el especial interés por analizar los usos y formas de consumo en el ciberespacio entre el colectivo adolescente, y su conexión con los problemas y riesgos que estas prácticas pueden acarrear. En general, el universo de estudio se ha movido en un arco de edad que se extiende entre los 10 y los 18 años, con pequeñas variaciones alrededor de este rango. A este respecto, debemos recalcar que este periodo de la vida se constituye como un momento especialmente destacado en el proceso de construcción de la identidad y en el que se manifiestan formas de comportamiento cualitativamente distintas de las etapas anteriores en lo que se refiere a las relaciones e interacciones con los otros.

3. Las relativamente escasas investigaciones en que se han combinado ambas metodologías y técnicas y que solo en algún caso abarcan el conjunto del territorio nacional. En cuanto a la forma de articular ambas metodologías, éstas se han abordado de manera sumatoria más que integradora, generando informes independientes. La complementariedad real de las metodologías cuantitativas y cualitativas proporciona la posibilidad de generar aproximaciones holísticas para el análisis y la interpretación de los datos, haciendo un uso más flexible de las técnicas de investigación donde los resultados de un método nos servirían como base para el siguiente. En este sentido, la investigación cualitativa puede servir como estudio exploratorio para establecer las hipótesis que serán recogidas en la parte cuantitativa para su verificación o refutación posterior. Paralelamente, el análisis cualitativo permitirá interpretar los resultados del análisis cuantitativo y facilitará una visión de la complejidad e interconexión de los diferentes aspectos que intervienen en el fenómeno estudiado.

4. Cabe subrayar el recurso de los investigadores a los centros educativos como lugares desde los que reclutar a los individuos que actuarán como unidades respondentes (la LOE -Ley Orgánica de Educación- considera la educación obligatoria desde los 6 a los 16 años, edad esta última que corresponde teóricamente con el cuarto y último curso de la ESO, aunque la ley también indica que los 
alumnos que no se gradúen en ESO a los 16 años tienen derecho a permanecer en régimen ordinario hasta los 18 años cursando dichos estudios) en la recolección/producción de datos primarios, ya sean cuantitativos o cualitativos. Las ventajas de esta práctica son evidentes respecto a las facilidades que ofrecen para acceder a los menores: se trata de grupos localizados en el tiempo y el espacio, permite acceder a algún tipo de listado de los que extraer a los sujetos seleccionados y facilita la obtención de los permisos paternos para acceder a los menores si éstos "cuentan con que el proceso es supervisado por las autoridades a cuyo cargo están estos grupos” (Rodríguez Pascual, 2006: 74).

5. En los estudios cuantitativos esta circunstancia se manifiesta en el predominio en los muestreos probabilísticos polietápicos por conglomerados, que en alguna de sus fases se corresponde con los centros educativos, en los cuales los adolescentes -las unidades muestrales últimas- están agrupados de manera natural. Al contrario de lo que sucede con los estratos, en el muestreo por conglomerados se busca hetereogeneidad al interior de los estratos y homogeneidad entre los mismos (Rodríguez Osuna, 1994). Los colegios cumplen este requisito en la medida que aglutinan alumnos y alumnas de diversas edades dentro del intervalo de edad objeto de nuestro estudio. Por su parte, la disparidad relativa al estatus socioeconómico se solventa estratificando por titularidad del centro, público y privado-concertado.

6. La selección de los colegios como unidades de muestreo primarias y no directamente a los individuos aporta notables ventajas en el proceso de selección de la muestra, disminuyendo los costes y el tiempo de ejecución del trabajo de campo. Los muestreos por conglomerados facilitan la elaboración del marco muestral porque permite el acceso a nuestra población objeto de estudio sin la necesidad de obtener un listado general de todos los niños de esas edades (listado que probablemente ni siquiera exista); basta con tener un listado de todos los centros educativos que cubren esa franja de edad del cual se seleccionarán los que formarán parte de la muestra (Pérez, 2009; Rodríguez Osuna, 1994).

Otra de las virtudes de este modelo de muestreo reside en que las muestras por conglomerados están concentradas frente a la dispersión de otros tipos de muestreo, por lo que simplifican el proceso de extracción de la muestra y disminuyen la necesidad de desplazamiento (Pérez, 2009). Teniendo en cuenta que a esas edades los individuos ya poseen una capacidad lectora-compresiva adecuada para cumplimentar el cuestionario sin la ayuda de un adulto, en estas investigaciones resulta habitual hacer uso del cuestionario autoadministrado, ya sea impreso o vía telemática, permitiendo que un grupo amplio de adolescentes cumplimenten el cuestionario simultáneamente en el aula con el asesoramiento de un entrevistador adiestrado; lo que permite, aún más, reducir el tiempo de ejecución y los costes asociados. 
7. Por el contrario, este tipo de muestreo tiene el inconveniente de aumentar el error muestral con respecto a un muestreo aleatorio simple para un tamaño dado de la muestra, debido a lo que Kish (1972) llama "efecto del diseño", que relaciona la varianza del conglomerado con la del muestreo aleatorio simple. Para disminuir el error muestral conviene ampliar el número de conglomerados (centros educativos) en la primera fase del muestreo, consiguiendo así disminuir la varianza entre conglomerados (Rodríguez Osuna, 2004).

El recurso a la estratificación por Comunidades Autónomas, titularidad del centro y niveles educativos también generarán un aumento de la precisión para la estimación global, compensando los posibles incrementos en el error estándar del muestreo por conglomerados. Pues "al dividir una población heterogénea en estratos homogéneos, el muestreo en estos estratos tiene poco error debido precisamente a la homogeneidad. El error total derivado del muestro en todos los estratos se observa que es menor que en el caso de no estratificar la población" (Pérez, 2009: 169). En efecto, la disminución en el error muestral del muestreo estratificado frente al muestreo aleatorio simple, y la correspondiente ganancia en precisión, ha sido ampliamente tratada en la literatura sobre el tema.

Finalmente, se ha visto el crecimiento en el número de estudios sobre este tema en España, que están en sintonía, desde un punto de vista metodológico, con lo que realiza en la esfera internacional. También, en nuestro trabajo, se ha apostado por la necesidad de tratar esta cuestión con criterios alejados de una visión negativa de la tecnología, evitando además un planteamiento estrictamente "adultocéntrico" que impida, en determinadas cuestiones, advertir certeramente los fenómenos que estamos estudiando.

\section{Referencias bibliográficas}

Aboujaoude, E; Koran, L.M.; Gamel, N.; Large, M. D. and Serpe, R.T. (2006): “Potencial markers for problematic internet use: a telephone survey of 2.515 adults", CNS Spectrum, no 11 , pp. 750-755.

ACPI-Protégeles, (2002): Seguridad infantily costumbres de los menores en Internet, Informe. En http://www.protegeles.com/internet.doc.

Albero, M. (2002): “Adolescentes e Internet. Mitos y realidades”, Zer, n.13. En: http://www.ehu.es/zer/zer13/adolescentes13.htm

Aguaded-Gómez, J. I. (2011): “Niños y adolescentes: nuevas generaciones interactivas”, Revista Comunicar, n 36, pp 7-8. 
Aranda, D.; Sánchez Navarro, J.; Tabernero, C. y Tubella, I. (2009): Jóvenes y ocio digital. Informe sobre el uso de herramientas digitales por parte de los adolescentes en España. Barcelona: UOC. En: http://spider-uoc.blogspot.com

Aranda, D.; Sánchez Navarro, J.; Tabernero, C. y Tubella, I. (2010): “Los Jóvenes del Siglo XXI: Prácticas Comunicativas y Consumo Cultural", II Congreso Internacional AE-IC "Comunicación y desarrollo en la era digital", Málaga 2010. En: http://www.aeic2010malaga.org/upload/ok/204.pdf

Ararteko (2009): La transmisión de valores a menores. Informe extraordinario de la Institución del Ararteko al Parlamento Vasco. En: http://s.libertaddigital.com/doc/informe-del-defensor-del-pueblo-y-unicef-41168033.pdf.

Aslanidou, Sofia and Menexes, George (2008): "Youth and the Internet: Uses and practices in the home", Computers \& Education, n. 51, pp. 1375-1391

Balbín Vich, F. de A. (2009): "Estudio de uso problemático de las tecnologías de la información, la comunicación y el juego entre adolescentes y jóvenes de la ciudad de Madrid". Trastornos adictivos: Órgano Oficial de la Sociedad española de Toxicomanías, vol. 11, n. 3, pp. 151-162.

Blanco, Ignacio y Römer, Max (ed.) (2010): Los niños frente a las pantallas. Madrid: Editorial Universitas.

Borges, J. L. (1981): El Hacedor. Madrid: Alianza.

Bringué Sala, X. y Sádaba Chalezquer, Ch. (2009): La generación Interactiva en España. Niños y jóvenes ante las pantallas. Madrid: Fundación Telefónica. En: http://www.fundación.telefonica.com/.../generacionesinteractivas.pdf/

Bringué Sala, X. y Sádaba Chalezquer, Ch. (2011): Menores y redes sociales. Madrid: Fundación Telefónica. http://www.generacionesinteractivas.org/wp-content/uploads/2011/01/Libro-Menores-y-Redes-Sociales_Fin.pdf

Burawoy, M. (2003): Dos métodos en pos de la ciencia. Skocol versus Trotski. Buenos Aires: Prometeo.

Callejo, J. (2004): “Limitaciones para el establecimiento de una tipología de los niños y la televisión: el caso español”, Revista de Metodología de Ciencias Sociales, n. 8, pp. 11-54.

Caplan, S. E. (2003): "Preference for online social interaction: A theory of problematic Internet use and psychosocial wellbeing”, Communication Research, vol. 30, n. 6, pp. 625-648.

Castellana Rosell, M.; Sánchez-Carbonell, Chamarro Lusar, A.; Graner, P. y Beranuy Fargues, M., (2007): Projecte d'investigació sobre les noves tecnologies i les addiccions dels adolescents envers aquesta temàtica. Barcelona: FPCEE-Blanquerna. Universitat Ramon Llull. En: http://internetsegura.udl.cat/images/4/41/Noves_addiccions_adolescencia.pdf

Chai, S. et al. (2009): “Internet and online information privacy: an exploratory study of preteens and early teens", IEE Transactions on Professional communication, vol. 52, n. 2.

Charlton, J. R. (2002): “A factor-analytic investigation of computer addiction and engagement", British Journal of Psycho$\log y$, n. 93, pp. 329-344. 
Charlton, J. R. and Danforth, I. D. W. (2007): “Distinguishing addiction and high engagement in the context of online game playing”, Computers in Human Behavior, vol. 23, pp. 1531-1548.

Conde, E., Ruiz, C. y Torres-Lana, E.: Relación entre el uso de Internet y el rendimiento académico en una muestra de adolescentes canarios. En http://www.gabinetecomunicacionyeducacion.com [Fecha de consulta: agosto 2010]

Davis, R. A. (2001): “A cognitive-behavioral model of pathological Internet use", Computer in Human Behavior, vol. 17, pp. 187-195.

Defensor del Menor y ACPI-Protégeles (2004): Cibercentros y Seguridad Infantil en Internet. Informe. En http://www.protegeles.com/cibercentro.asp

Defensor del Pueblo (2010): Programación y contenidos de la televisión e Internet: la opinión de los menores sobre la protección de sus derechos. Madrid: Informes, estudios y documentos; 25, Madrid. En: www.defensordelpueblo.es/es/ Documentacion/Publicaciones/monografico/index.html

Echeburúa, E.; Labrador, F. J. y Becoña, E. (2009): Adicción a las nuevas tecnologías en adolescentes y jóvenes. Madrid: Pirámide.

Espinar Ruiz, E. y González Río, M. J. (2008): "Jóvenes conectados. Las experiencias de los jóvenes con las nuevas tecnologías”, RES. Revista Española de Sociología, n. 9, pp.109-122.

Fuente Cobo, C. y Ruiz San Román, J. A. (2011): "Protección de la infancia en la nueva regulación audiovisual en España", Revista Latina de Comunicación Social, 66, pp. 153-177.

Fundación Kaiser (2009): Generation M2: Media in the lives of 8 to 18 years old". En: http:/ /www.kff.org/entmedia/upload/ 8010 [consultado el 24 de febrero de 2010].

Fundación Pfizer (2009): La Juventud y las Redes Sociales en Internet. Informe de resultados de la encuesta. En: www.fundacionpfizer.org

García Galera, Ma . C. (2008): “Los derechos de la Infancia frente al televisor, ¿Quién los defiende?”, Doxa Comunicación, nº 6, pp. 93-111.

García Jiménez, A. et al. (2010a): "Los menores y los usos de Internet: Riesgos y características", Congreso AEIC 2010 "Comunicación y desarrollo en la era digital", Málaga 2010. En: http://www.aeic2010malaga.org/upload/ok/315.pdf

García Jiménez, A. (Coord.) (2010b): Comunicación y comportamiento en el ciberespacio. Actitudes y riesgos de los adolescentes. Barcelona: Icaria.

Garitaonandia, C. y Garmendia, M. (2007): “Cómo Usan Internet Los Jóvenes: Hábitos, Riesgos y Control Parental”. Informe Eukidsonline. En: www.ehu.es/eukidsonline/INFORME\%20FINAL-INTERNET.pdf [Consultado en agosto de 2010].

Garmendia, M. et al. (2011): Riesgos y seguridad en Internet: los menores españoles en el contexto europeo. En: www.ehu.es/eukidsonline 
Gertrudix Barrio, Manuel y Gertrudix Barrio, Felipe (2007): “Investigaciones en torno a las tic en educación: una panorámica actualizada”. Docencia e Investigación: revista de la Escuela Universitaria de Magisterio de Toledo, año 32, n 17, pp. $119-146$

Gil, A.; Feliu, J.; Rivero, I. y Gil, E. P. (2003): “¿Nuevas tecnologías de la información y la comunicación o nuevas tecnologías de relación? Niños, jóvenes y cultura digital”. UOC. En http://www.uoc.edu/dt/20347/index.html [Consultado en agosto de 2010]

Graner Jordana, C.; Castellana Rosell, M.; Sánchez-Carbonell, X.; Beranuy Fargues, M. y Chamarro Lusar, A. (2006): “Comparación entre el uso de Internet por parte de adolescentes y jóvenes". XXI Congreso Internacional de Comunicación: Los jóvenes y los nuevos escenarios de comunicación, Pamplona: Universidad de Navarra. En: http://www.unav.es/fcom/ cicom/2006/es/grupostrabajo.htm

Graner, P.; Beranuy Fargues, M.; Sánchez-Carbonell, X.; Chamarro Lusar, A. y Castellana Rosell, M. (2007): “¿Qué uso hacen los jóvenes y adolescentes de Internet y del móvil?”, en Alvarez Pousa, L.; Evans Pim, J. y Crespo Argibay, O. (ed. lit.): Comunicación e xuventude: Actas do Foro Internacional, pp. 71-90. En: http://dialnet.unirioja.es/servlet/articulo? codigo $=2648887$

Greenfield, D.N. (1999a): Virtual addiction. Oakland, CA: New Harbinger Publications.

- (1999b): “The Nature of Internet Addiction: Psychological Factors in Compulsive Internet Use”, American Psychological Association Convention.

Hernández Prados, M. A.; Sánchez Vera, M. M. y Fernández, M. I. (2006): “Las tics, inuevos medios o medios conocidos para los Jóvenes?”, XXI Congreso Internacional de Comunicación: Los jóvenes y los nuevos escenarios de comunicación, Pamplona: Universidad de Navarra, 9 y 10 de noviembre de 2006. En: http://www.unav.es/fcom/cicom/2006/es/ grupostrabajo.htm

Hundley, H. L. and Shyles, L. (2010): “Us teenagers' perceptions and awareness of digital technology: a focus group approach”, New Media \& Society, vol. 12, n. 3, pp. 417-433.

Ibáñez. J. (1994): "Perspectivas de la investigación social: el diseño de las tres perspectivas”, en García Ferrando, M., Ibáñez, J. y Alvira, F. (comp.): El análisis de la realidad social. Métodos y técnicas de investigación. Madrid: Alianza Editorial.

Kish, L. (1972): Muestreo de encuestas. México: Trillas.

Lenhart, A. et. al. (2010): "Social media \& Mobile Internet Use Among Teens and Young Adults", Pew Internet \& American. En: http://pewinternet.org/ /media//Files/Reports/2010/PIP_Social_Media_and_Young_Adults_Report_Final_with_toplines.pdf

Livingstone, S. \& Haddon, L. (2008): "Risky experiences for children online: charting European research on children ant the Internet”, Children \& Society, vol. 22, pp. 314-323. 
López Caballero, A. (2009-2010): “Los adolescentes y las redes sociales en Internet”, Diálogo Familia Colegio, n. 281, pp. 310.

Llorca, M. A. (2009): Hábitos y uso de los videojuegos en la comunicación visual: influencia en la inteligencia espacial y el rendimiento escolar. Granada: Editorial Universidad de Granada. Tesis doctoral.

Madruga Vicente, M.; Prieto Prieto, David, J.; Cerro Herrero, D. y Espínola Pardo, M. T. (2005): "Hábitos de vida relacionados con la salud y uso de Internet en adolescentes extremeños”, en Hermoso Ruiz, F. (coord.). VIII Congreso de Estudios Extremeños, Libro de Actas. Diputación de Badajoz, pp. 1351-1367.

Meerkek, G.; Van den Eijinden, R. and Garretsen, H. (2006): "Predicting compulsive Internet use: it’s all aboutsex”, Cyperpsychology \& Behavior, vol. 9, pp. 95-103.

Muñoz-Rivas, M. J., Navarro, M. E: y Ortega, N. (2003): "Patrones de uso de Internet en población universitaria española", Adicciones, n. 15, pp. 137-144.

Mut Amengual, Tomeu Morey López, M. (2008): "Preferencias en el uso de internet, televisión, videoconsolas y teléfonos móviles entre los menores de las Islas Baleares”, Edutec: Revista electrónica de tecnología educativa, n. 27. En: http://edutec.rediris.es/Revelec2/revelec27/articulos_n27_PDF/Edutec-E_Mut_Morey_n27.pdf

Navarro Córdoba, L. F. (2003): “Los hábitos de consumo en medios de comunicación en los jóvenes cordobeses”, Comunicar: Revista científica iberoamericana de comunicación y educación, n. 21, pp. 167-171. En: http://dialnet.unirioja.es/ servlet/fichero_articulo?codigo=755255

Núñez Ladevéze, L; Cantavella, J. y Vázquez Barrio, T. (2008): "Spanish Children as captive market of screens”, Newsletter on Children, Youth and Media in the World, $\mathrm{n}^{\circ}$ 1, pp. 1-13.

Observatorio de la Infancia en Andalucía (2008): Nuevas tecnologías e infancia y adolescencia 2008, Informe. Sevilla: Junta de Andalucía, Consejería de Innovación, Ciencia y Empresa, Consejería para la Igualdad y Bienestar Social. En: http://www.juntadeandalucia.es/observatoriodelainfancia/OIA/esp/descargar.aspx?id=1969\&tipo=documento

Observatorio de la Infancia de Andalucía (2010): Actividades y usos de las TIC entre los chicos y las chicas en Andalucía. Informe 2010. Sevilla: Junta de Andalucía, Consejería de Innovación, Ciencia y Empresa, Consejería para la Igualdad y Bienestar Social. En: http://www.juntadeandalucia.es/observatoriodelainfancia/oia/esp/documentos_ficha.aspx?id=2734

Observatorio de la Seguridad en la Información (2009): Estudio sobre hábitos seguros en el uso de las TIC por niños y adolescentes y e-confianza de sus padres. León: Instituto Nacional de Tecnologías de la Comunicación (INTECO).

Olson, C.K., Kutner L., \& Beresin, E.V. (2007): “Children and video games: How much do we know?”, Psychiatric Times, vol. 24, n. 12. En: http://www.psychiatrictimes.com/display/article/10168/54191?verify=0

Pérez, C. (2009): Técnicas de muestreo estadístico. Madrid: Garceta. 
Ramírez, O. J. (2009): "Riesgos de origen tecnológico: apuntes conceptuales para una definición, caracterización y reconocimiento de las perspectivas de estudio del riesgo tecnológico”, Luna Azul, no 29, julio-diciembre 209, pp. 82-94.

Ramírez de la Piscina, T. et al. (2006): "Estudio sobre la alfabetización audiovisual de los adolescentes vascos”, Zer, n. 21, pp. 177-202. En: http://www.ehu.es/zer/

Red.es (2006): Infancia y adolescencia en la Sociedad de la Información. Análisis de la relación con las TIC en el hogar. Ministerio de Industria, Turismo y Comercio. En: http://www.red.es

Rodríguez Osuna, J. (1994): “La muestra: teoría y aplicación”, en García Ferrando, M., Ibáñez, J. y Alvira, F. (comp.): El análisis de la realidad social. Métodos y técnicas de investigación. Madrid: Alianza Editorial.

Rodríguez Pascual, I. (2006): "Redefiniendo el trabajo metodológico cualitativo con niños: el uso de la entrevista de grupo aplicada al estudio de la tecnología”, Revista de Metodología de Ciencias Sociales, n. 12, pp. 65-88. En: http://dialnet.unirioja.es/servlet/articulo?codigo $=2216545$

Rodríguez Pascual, Iván (2010): "E-Generaciones: ¿̨uánto hay de Adultocéntrico en el Análisis de la Relación entre la Población Infantil y las Nuevas Tecnologías?”, Intervención Psicosocial, vol. 19, n. 1, pp. 9-18.

Rubio, A. (dir.) (2009): Adolescentes y jóvenes en la red: factores de oportunidad. Injuve. En: http://www.injuve.migualdad.es:80/injuve

Sánchez Burón, A. y Fernández Marín, M. (2010): Generación 2.0, 2010. Hábitos de los adolescentes en el uso de las redes sociales. Universidad Camilo José Cela. En: http://www.slideshare.net/ucjc/generacin-20-hbitos-de-los-ad

Sánchez Burón, A.; Rodríguez Tapia, L.T. y Fernández Martín, P. (2009): Los adolescentes en la Red. Estudio sobre los hábitos de los adolescentes en el uso de Internet y redes sociales, Resumen Ejecutivo. Dept. de Psicología de la Universidad Camilo José Cela. En: www.tictarragona.com/.../los-adolescentes-en-la-red-estudio-sobre-sus-habitos-de-comportamiento-eninternet/ [consultado en agosto de 2010].

Sánchez Martínez, M. y Otero Puime, A. (2010): "Usos de internet y factores asociados en adolescentes de la Comunidad de Madrid-2", Atención primaria: Publicación oficial de la Sociedad Española de Familia y Comunitaria, vol. 42, n. 2, pp. 79-85

Siomos, K. E. et al. (2008) : “Internet Adiction among Greek Adolescent Students”, Cyberpsychology \& Behavior, vol. 11, n. 6, pp. 653-657.

Sureda, J. et al. (2008): Les TIC i els menors a les Illes Balears, Fundación Ille Balears Innovació Tecnològica y Observatori de la societat de la informació (edit). En: http://www.doredin.mec.es/documentos/01220092004487.pdf

Torrecilla Jiménez, J. M. (dir.) (2008): Estudio de uso problemático de las tecnologías de la información, la comunicación y el juego entre adolescentes y jóvenes de la ciudad de Madrid. Madrid: Instituto de Adicciones Madrid Salud. Documentos técnicos del Instituto de Adicciones de Madrid Salud. 
Viñas Poch, F. (2009): “Uso autoinformado de Internet en adolescentes: perfil psicológico de un uso elevado de la red”, International Journal of Psychology and Psychological Therapy, vol. 9, n. 1, pp. 109-122.

Viñas, F; Juan, J.; Villar, E.; Caparrós, B.; Perez, I. y Corrella, M. (2002): “Internet y psicopatología: las nuevas formas de comunicación y su relación con diferentes índices de psicopatología”, Clínica y Salud, vol. 13, pp. 25-256.

Young, K. S. (1996): "Psychology of computer use: XL Addictive use ot the Internet: A case that break the stereotype”, Psychological Reports, vol. 79, pp. 899-902.

Young, K. S. (1998): Caught in the net. New York: John Wiley \& Sons, Inc. 